\title{
ERCC1 rs11615 polymorphism and chemosensitivity to platinum drugs in patients with ovarian cancer: a systematic review and meta-analysis
}

\author{
Yuqiang Zhang ${ }^{1 \dagger}$, Sufen $\mathrm{CaO}^{1 \dagger}{ }^{\dagger}$, Chunyu Zhuang ${ }^{2}$, Jiacheng Chen ${ }^{3}$, Xiaojing Chen ${ }^{4}$, Hong Sun ${ }^{4}$,
} Shengying $\operatorname{Lin}^{5}$ and Bailang $\operatorname{Lin}^{2^{*}}$ (1)

\begin{abstract}
Objective: To explore the relationship between ERCC1 rs11615 polymorphism and chemosensitivity to platinum drugs in ovarian cancer by the method of meta-analysis.

Methods: Pubmed, Web of Science, EMBASE, Cochrane Library, China National Knowledge Infrastructure (CNKI), and China Wanfang databases were comprehensively searched up to September 2020, to identify the relationship between ERCC1 rs11615 polymorphism and chemosensitivity of ovarian cancer. The data was analyzed by Stata 15.0 statistic software.

Results: A total of 10 published papers were included, including 1866 patients with ovarian cancer. The results showed that compared allele $C$ at ERCC 1 rs 11615 locus with allele T, the pooled OR was 0.92 (95\%Cl:0.68 1.24, $P>0.05)$. There were no significant differences in recessive, dominant, homozygous, and heterozygous models. In accordance with a subgroup analysis of Ethnicity, all genotypes were statistically significant in the Asian population. In the allelic, dominant, recessive, homozygous and heterozygous models, the OR was 0.70 (95\%Cl:0.51 0.95), 0.20 (95\%Cl:0.07 0.56), 0.79 (95\%Cl:0.63 1.00), 0.21 (95\%Cl:0.07 0.59), 0.19 (95\%Cl:0.07 0.54), respectively, while in the Caucasian population, no statistically significant genotype was found.
\end{abstract}

Conclusion: The ERCC1 rs11615 polymorphism is associated with chemosensitivity in patients with ovarian cancer, especially in the Asian population, but not in the Caucasian population.

Keywords: Ovarian cancer, ERCC1, Platinum drugs, Meta-analysis

\section{Introduction}

The ovarian malignant tumor is one of the common malignant tumors of female reproductive organs, which is a severe threat to women's health. Its incidence is second only to cervical cancer and uterine cancer, and its

\footnotetext{
*Correspondence: linbailang123@126.com

†Yuqiang Zhang and Sufen Cao contributed equally to this work.

${ }^{2}$ Department of Nursing, Haikou Maternal and Child Health Hospital, No. 6 Wentan Road, Haikou 570203, Hainan, China

Full list of author information is available at the end of the article
}

mortality ranks first among gynecological malignant tumors [1]. According to the latest data, there are about 300,000 cases of female ovarian cancer each year worldwide, with a death rate of 185,000 and a mortality rate of $0.45 \%$ [1]. Due to ovarian cancer's insidious onset and lack of early diagnostic indicators, most of them are diagnosed in the late stage. The pathogenic mechanism of ovarian cancer is obscure, and it is generally believed to be the result of heredity and environment $[2,3]$. At present, platinum-based combination chemotherapy is the 
first-line treatment for ovarian cancer after cytoreductive surgery. However, in the initial treatment period, $20 \%$ of patients do not respond to platinum chemotherapy, and up to $75 \%$ of patients with ovarian cancers have a relapse. Meanwhile, patients with the treatment after the relapse tend to have resistance to platinum drugs $[4,5]$. If the drug resistance of ovarian cancer patients can be predicted before or at the initial stage of chemotherapy in order to adjust the chemotherapy scheme in time, the tumor remission rate can be enhanced, and the prognosis of patients can be improved $[6,7]$. Therefore, identifying the sensitivity of patients to platinum drugs is the key to effectively enhance the efficacy of chemotherapy for ovarian cancer. Platinum drugs have been the most widely used chemotherapeutic drugs since they were put into clinical application in the 1970s [8, 9]. Clinically, platinum drugs have been extensively used in the treatment of malignant tumors such as nasopharyngeal carcinoma [10], esophageal cancer [11], breast cancer [12], etc. Therefore, platinum drugs have become a symbol of an era among anticancer drugs [13]. Platinum compounds (carboplatin or cisplatin) exert their antitumor effect through combining with DNA to form intrachain and interchain crosslinking and Pt-DNA adducts [14]. DNA adducts cause structural changes in DNA, which affects DNA replication and inhibits DNA synthesis. There are many factors in the development of tumor cells to platinum resistance, which may include a decrease of drug accumulation, an increase of glutathione level and metallothionein, and an improvement of DNA repair ability [15]. However, it is believed that DNA repair is the fundamental contributor to clinical drug resistance in platinum-based therapy, and the difference in individual DNA repair ability is closely related to platinum prognosis in patients with ovarian cancer [5]. Moreover, studies have confirmed that the clinical remission rate of tumor patients is related to the level of Pt-DNA adducts in their blood circulation [16]. If the DNA repair ability of tumor cells is reduced, the clearance of Pt-DNA adducts in blood circulation will be diminished, which leads to the augmented curative effect of platinum chemotherapeutic drugs. Otherwise, the curative effect will be unsatisfactory $[16,17]$. Therefore, the DNA repair ability is the main factor that affects the efficacy of platinum drugs.

Nucleotide excision repair (NER) is one of the classic ways to participate in multi-drug resistance in ovarian cancer [18]. As an essential member of this pathway, ERCC1 gene expression products exert such function to recognize DNA damage in the initial repair stage [19]. Platinum-based chemotherapeutics take the role of an anti-cancer way mainly by cross-linking cancer cell DNA [18]. Previous studies have shown that changes in the third base $(19007 \mathrm{C} / \mathrm{T})$ of the fourth exon of the ERCC1 gene can reduce its gene transcription activity by $50 \%$, leading to a decrease in its protein or mRNA expression and affect chemosensitivity [20]. Therefore, the $19007 \mathrm{C} / \mathrm{T}$ polymorphism of the ERCC1 gene is theoretically related to the sensitivity of platinum-based combined chemotherapy. However, the conclusions of previous studies on the ERCC1 rs11615 polymorphism are not consistent. Steffensen et al. [21] showed that the chemotherapeutic responses of the ovarian cancer patients with genotype TT at ERCC1 rs11615 locus were better than those with genotype TC $+\mathrm{CC}$, while Kang et al. [22] believed that ovarian cancer patients with genotype $\mathrm{CT}+\mathrm{TT}$ were more sensitive to platinum chemotherapy than those with genotype CC. Therefore, we systematically reviewed the related literature in this study, applying meta-analysis to conduct a comprehensive analysis upon the relationship between the ERCC1 rs11615 polymorphism and the chemosensitivity of platinum drugs in patients with ovarian cancer.

\section{Methods \\ Literature retrieval}

Pubmed, Web of Science, EMBASE, Cochrane Library, China National Knowledge Infrastructure (CNKI), and China Wanfang databases were searched for literature about ERCC1 rs11615 polymorphism and chemosensitivity of ovarian cancer, from inception to September 2020. The search strategy was as follows: ("ERCC1" OR "excision repair cross-complementation group 1") AND ("ovarian carcinoma" OR "ovarian cancer" OR "ovarian tumor") AND ("polymorphism" OR "gene mutation". There was no language limitation. Two researchers independently searched and cross-checked the literature. When the two researchers disagreed with each other, they resolved disagreements through discussion, or turned to a third researcher for verification.

\section{Inclusion and exclusion criteria Inclusion criteria}

(1) patients with ovarian cancer confirmed by pathological examination; (2) patients received platinum-based combined chemotherapy after operation; (3) genotypes were detected by genomic DNA extracted from tumor tissue or peripheral blood; (4) studies with a large number of cases were selected if the same samples were used in multiple articles; (5) the target gene polymorphism was ERCC1 rs11615 locus or $19007 \mathrm{C} / \mathrm{T}$.

\section{Exclusion criteria}

(1) reviews, letters and conference summaries, etc.; (2) repeated reports, insufficient data, too little reported information, and unavailable literature; (3) studies with Newcastle-Ottawa scale (NOS) scores less than 6. 


\section{Literature quality evaluation}

The quality of the included studies was evaluated according to NOS [23], which was carried out independently by two researchers, and finally cross-checked. When there was a disagreement, it was resolved through discussion, or it was decided by a third researcher.

\section{Data extraction}

All literature data were extracted independently by two researchers and finally cross-checked. The following information was extracted from each included literature: author, year of publication, country of the first author, patient ethnicity, total number of cases, chemotherapy regimen, genotype detection methods, and the number of patients with genotype sensitivity and drug resistance.

\section{Statistical analysis}

The data were analyzed by Stata 15.0 statistical software. The odds ratio (OR) and its $95 \% \mathrm{CI}$ were calculated as effect size. Q-test was used to assess the heterogeneity across the studies included. If $\mathrm{I}^{2} \geq 50 \%$, or $P \leq 0.05$, the heterogeneity was considered to exist, and thus a random-effects model (REM) was adopted. If $\mathrm{I}^{2}<50 \%$ and $P>0.05$, the heterogeneity was considered to be not significant, so a fixed-effects model (FEM) was used for data merging. If there was heterogeneity, subgroup analysis was carried out according to Ethnicity and Hardy-Weinberg Equilibrium (HWE) to explore the source of heterogeneity. Z-test was used to test the significance of the pooled OR value. Funnel plot and Egger's Test were used to evaluate publication bias. The funnel plot was made by filling and trimming methods. Finally, a sensitivity analysis was conducted to verify the robustness of the research results.

\section{Results}

\section{Features of the included studies}

According to the strict inclusion criteria, a total of 10 articles were included [21, 22, 24-31], including a total of 1866 patients with ovarian cancer. The specific literature screening process is shown in Fig. 1, and the basic characteristics of the included studies are shown in Table 1, from which it can be seen that the NOS scores of the included studies are all above 6 .

\section{The results of meta-analysis Allele comparison}

The main results of the meta-analysis were shown in Table 2 and Fig. 2. The heterogeneity test results showed that $\mathrm{I}^{2}=66.4 \%(P<0.05)$, which indicated significant differences in heterogeneities between studies, so the REM was adopted. The forest plot was shown in Fig. 2A. Compared with allele $\mathrm{T}$, the chemosensitivity to platinum drugs of allele C of ERCC1 rs11615 was set as control. The results showed that OR $=0.92$ (95\%CI:0.68, 1.24, $P>0.05)$. Ethnic subgroup analysis showed that for the Asian population, OR $=0.70$ (95\%CI:0.51 0.95), the difference was statistically significant, and the heterogeneity

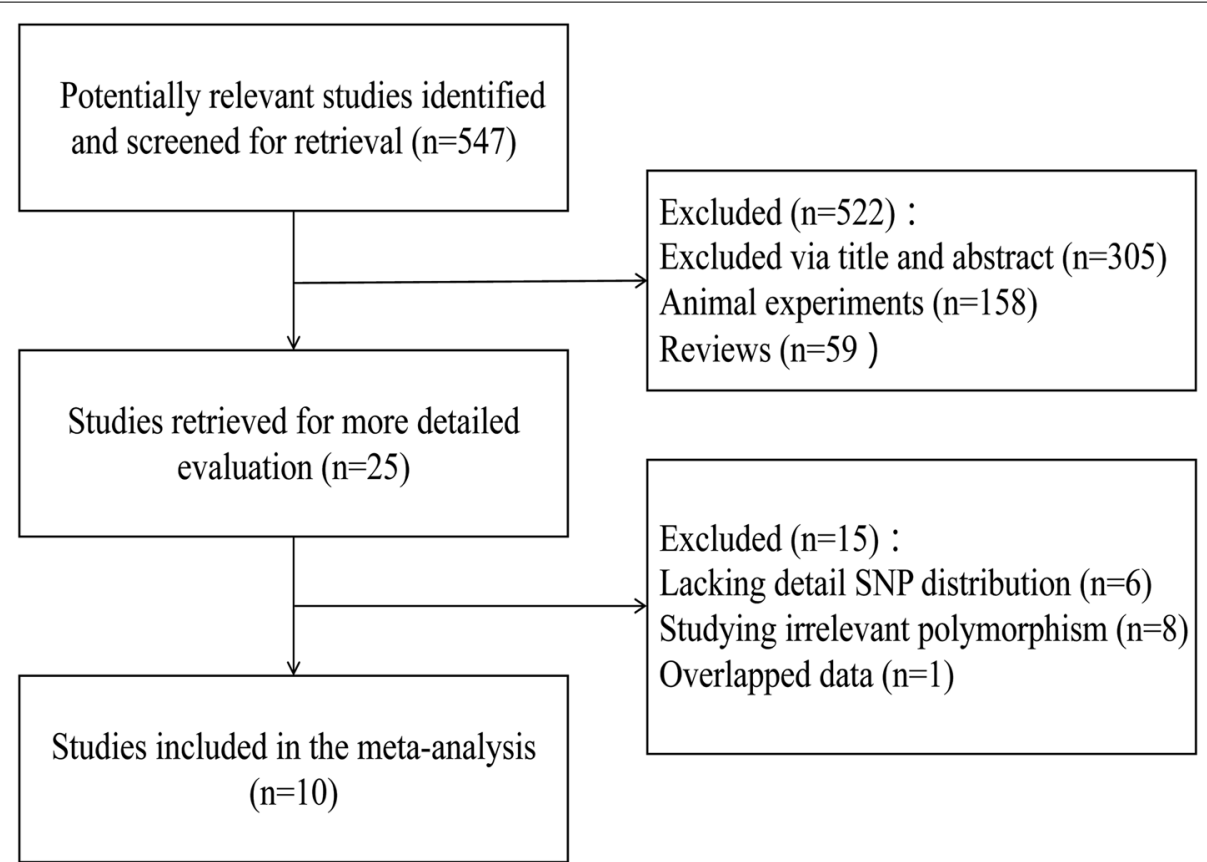

Fig. 1 A flow diagram of the study selection process 


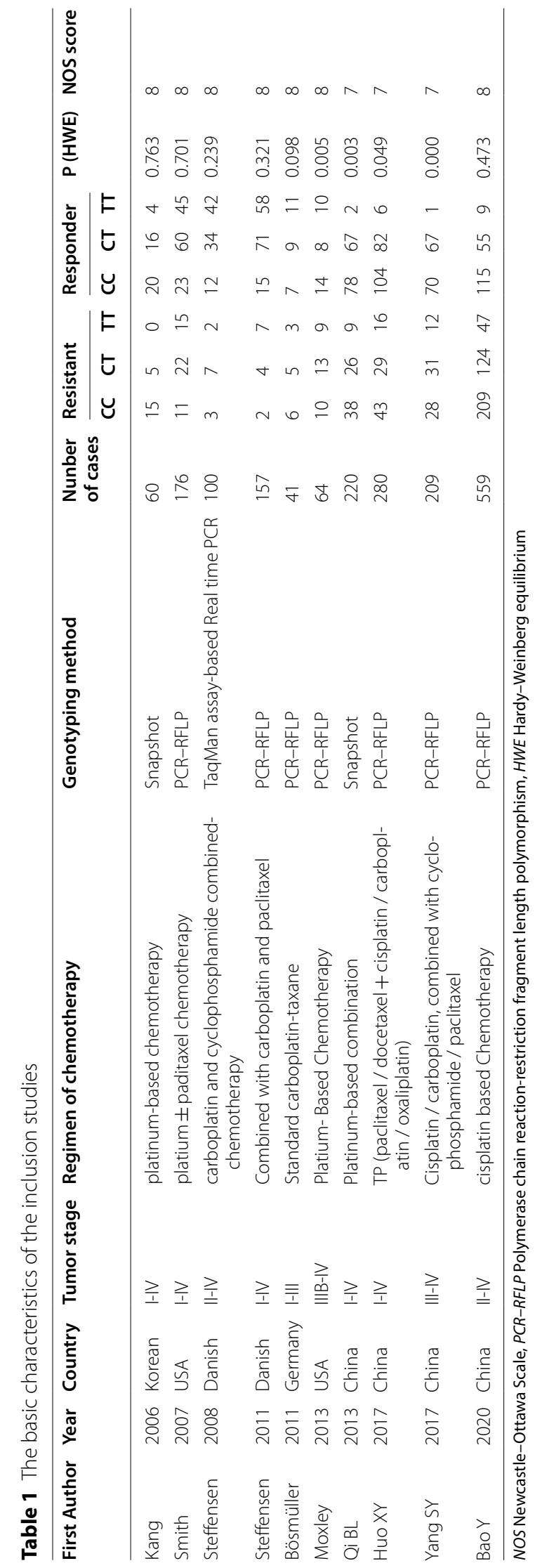


Table 2 Meta-analysis of ERCC1 rs11615 polymorphism and chemosensitivity of ovarian cancer (subgroup analysis by ethnicity and HWE)

\begin{tabular}{|c|c|c|c|c|c|c|c|c|c|}
\hline Genetic models & Subgroup & $n$ & OR & $95 \% \mathrm{Cl}$ & $P_{r}$ & $\mathrm{I}^{2}(\%)$ & $P_{h}$ & Model & $P_{b}$ (Egger's Test) \\
\hline C vs.T & Overall & 10 & 0.92 & $0.68 \sim 1.24$ & 0.574 & 66.4 & 0.002 & REM & 0.006 \\
\hline \multirow[t]{2}{*}{ Ethnicity } & Asian & 5 & 0.70 & $0.51 \sim 0.95$ & 0.021 & 57.7 & 0.051 & REM & 0.048 \\
\hline & Caucasian & 5 & 1.26 & $0.85 \sim 1.85$ & 0.246 & 29.0 & 0.228 & FEM & 0.463 \\
\hline \multirow[t]{2}{*}{ HWE } & Yes & 7 & 1.12 & $0.73 \sim 1.73$ & 0.601 & 73.9 & 0.001 & REM & 0.014 \\
\hline & No & 3 & 0.65 & $0.49 \sim 0.87$ & 0.003 & 0.0 & 0.433 & FEM & 0.557 \\
\hline $\mathrm{CC}+\mathrm{CT}$ vs.TT & Overall & 10 & 0.59 & $0.27 \sim 1.31$ & 0.194 & 77.0 & 0.000 & REM & 0.165 \\
\hline \multirow[t]{2}{*}{ Ethnicity } & Asian & 5 & 0.20 & $0.07 \sim 0.56$ & 0.002 & 63.6 & 0.027 & REM & 0.315 \\
\hline & Caucasian & 5 & 1.32 & $0.75 \sim 2.33$ & 0.342 & 24.1 & 0.261 & FEM & 0.221 \\
\hline \multirow[t]{2}{*}{ HWE } & Yes & 7 & 0.87 & $0.36 \sim 2.08$ & 0.750 & 75.9 & 0.000 & REM & 0.060 \\
\hline & No & 3 & 0.18 & $0.02 \sim 1.63$ & 0.127 & 83.9 & 0.002 & REM & 0.260 \\
\hline CC vs. CT+TT & Overall & 10 & 0.85 & $0.69 \sim 1.05$ & 0.138 & 28.7 & 0.181 & FEM & 0.011 \\
\hline \multirow[t]{2}{*}{ Ethnicity } & Asian & 5 & 0.79 & $0.63 \sim 1.00$ & 0.046 & 38.7 & 0.163 & FEM & 0.052 \\
\hline & Caucasian & 5 & 1.23 & $0.74 \sim 2.05$ & 0.416 & 0.0 & 0.501 & FEM & 0.351 \\
\hline \multirow[t]{2}{*}{ HWE } & Yes & 7 & 0.90 & $0.70 \sim 1.17$ & 0.432 & 44.0 & 0.098 & FEM & 0.010 \\
\hline & No & 3 & 0.75 & $0.52 \sim 1.10$ & 0.139 & 0.0 & 0.522 & FEM & 0.674 \\
\hline CC vs.TT & Overall & 10 & 0.62 & $0.26 \sim 1.48$ & 0.281 & 75.1 & 0.000 & REM & 0.054 \\
\hline \multirow[t]{2}{*}{ Ethnicity } & Asian & 5 & 0.21 & $0.07 \sim 0.59$ & 0.003 & 63.3 & 0.028 & REM & 0.279 \\
\hline & Caucasian & 5 & 1.51 & $0.83 \sim 2.73$ & 0.175 & 0.0 & 0.461 & FEM & 0.230 \\
\hline \multirow[t]{2}{*}{ HWE } & Yes & 7 & 1.04 & $0.39 \sim 2.82$ & 0.935 & 75.1 & 0.000 & REM & 0.014 \\
\hline & No & 3 & 0.16 & $0.02 \sim 1.08$ & 0.059 & 76.2 & 0.015 & REM & 0.305 \\
\hline CT vs. TT & Overall & 10 & 0.56 & $0.25 \sim 1.27$ & 0.163 & 74.4 & 0.000 & REM & 0.269 \\
\hline \multirow[t]{2}{*}{ Ethnicity } & Asian & 5 & 0.19 & $0.07 \sim 0.54$ & 0.002 & 62.1 & 0.032 & REM & 0.436 \\
\hline & Caucasian & 5 & 1.32 & $0.70 \sim 2.47$ & 0.392 & 24.0 & 0.262 & FEM & 0.280 \\
\hline \multirow[t]{2}{*}{ HWE } & Yes & 7 & 0.76 & $0.33 \sim 1.77$ & 0.529 & 70.3 & 0.003 & REM & 0.147 \\
\hline & No & 3 & 0.20 & $0.02 \sim 2.37$ & 0.201 & 85.8 & 0.001 & REM & 0.350 \\
\hline
\end{tabular}

OR Odds ratio, HWE Hardy-Weinberg equilibrium, REM Random-effects model, FEM Fixed-effects model, $P_{r} \mathrm{P}$ for OR, $P_{h} \mathrm{P}$ for Heterogeneity, $P_{b} \mathrm{P}$ for Publication bias

also decreased. While in the Caucasian population, the difference was not statistically significant. Subgroup analysis of HWE showed that there was no notable decrease in heterogeneity. The symmetry of the funnel plot was general (Fig. 3A), and Egger's Test showed that $P<0.05$, which indicated that there was a certain publication bias.

\section{Dominant genetic model}

In the dominant genetic model $(\mathrm{CC}+\mathrm{CT}$ vs. TT)), genotype $\mathrm{CC}+\mathrm{CT}$ was used as the exposure factor, genotype TT as the non-exposure factor, and chemosensitivity as the control. Heterogeneity test showed that $\mathrm{I}^{2}=77.0 \%(P<0.05)$, indicating the existence of the heterogeneity. Hence, a FEM model was adopted. The forest plot was as shown in Fig. 2B. The results showed that $\mathrm{OR}=0.59(0.27,1.31, P>0.05)$. The ethnic subgroup analysis showed that for the Asian population, $\mathrm{OR}=0.20$ (95\%CI:0.07 0.56), the difference was statistically significant. In the Caucasian population, the difference was not statistically significant. The heterogeneity decreased remarkably. The subgroup analysis of HWE showed that the decrease of heterogeneity was not obvious. The funnel plot was basically symmetrical (Fig. 3B), and Egger's Test showed that $P>0.05$, which indicated that there was no publication bias.

\section{Recessive genetic model}

In the recessive genetic model $(\mathrm{CC}$ vs. $\mathrm{CT}+\mathrm{TT})$, genotype $\mathrm{CC}$ was used as the exposure factor, genotype $\mathrm{CT}+\mathrm{TT}$ as the non-exposure factor, and chemosensitivity as the control. The heterogeneity test showed that $\mathrm{I}^{2}=28.7 \%(P>0.05)$, indicating that the heterogeneity across studies was not significant. Therefore, a FEM was used. The forest plot was as shown in Fig. 2C. The results showed that OR $=0.85$ (95\%CI:0.69 1.05, $P>0.05)$, which was consistent with the result of the subgroup analysis of HWN. The ethnic subgroup analysis showed that in the Asian population, $\mathrm{OR}=0.21$ (95\%CI: $0.07 \sim 0.59)$, the difference was statistically noteworthy. In the Caucasian population, the difference was not statistically meaningful. The subgroup analysis of HWE showed that there was no notable decrease in heterogeneity. The 

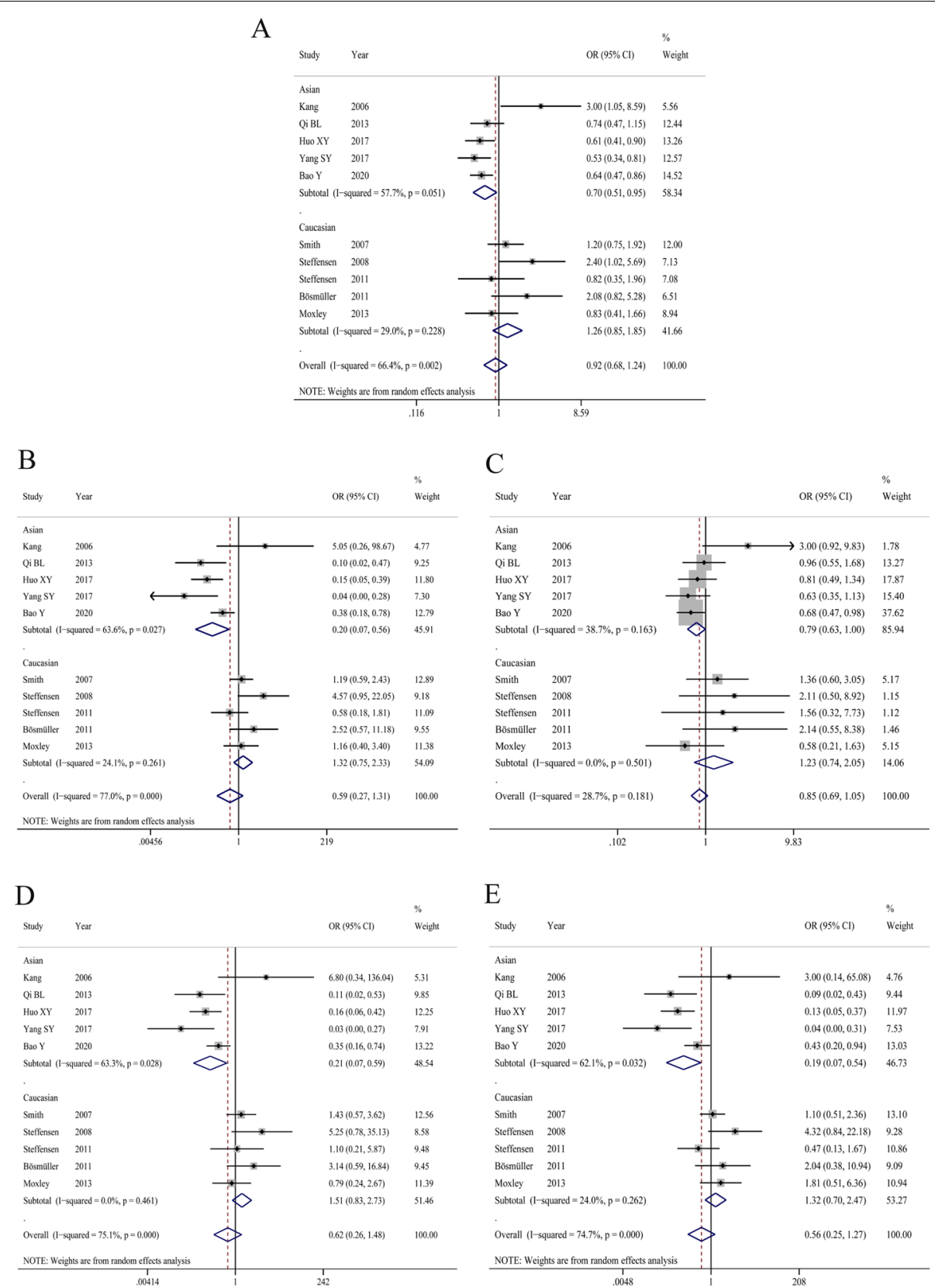

Fig. 2 Forest plots for the association between ERCC1 Rs11615 polymorphism and chemosensitivity to platinum drugs in ovarian cancer (A allele model; $\mathbf{B}$ dominant gene model; $\mathbf{C}$ recessive gene model; $\mathbf{D}$ homozygous model; $\mathbf{E}$ heterozygous model)

funnel plot was basically symmetrical (Fig. 3C), while Egger's Test showed that $P<0.05$, indicating a certain publication bias.

\section{Homozygous genetic model}

In the homozygous genetic model (CC vs. TT), genotype $\mathrm{CC}$ was used as the exposure factor, genotype TT as the non-exposure factor, and chemosensitivity as the control. The results of the heterogeneity test showed that $\mathrm{I}^{2}=75.1 \% \quad(P<0.05)$, indicating that there were noteworthy differences in heterogeneity across studies. Hence, a REM was adopted. The forest plot was as shown in Fig. 2D. The results showed that $\mathrm{OR}=0.62$ (95\% CI:0.26, 1.48, $P>0.05)$. The ethnic subgroup analysis showed that for the Asian population, $\mathrm{OR}=0.21$ (95\%CI: $0.07 \sim 0.59$ ), the difference was statistically significant. In the Caucasian population, the 


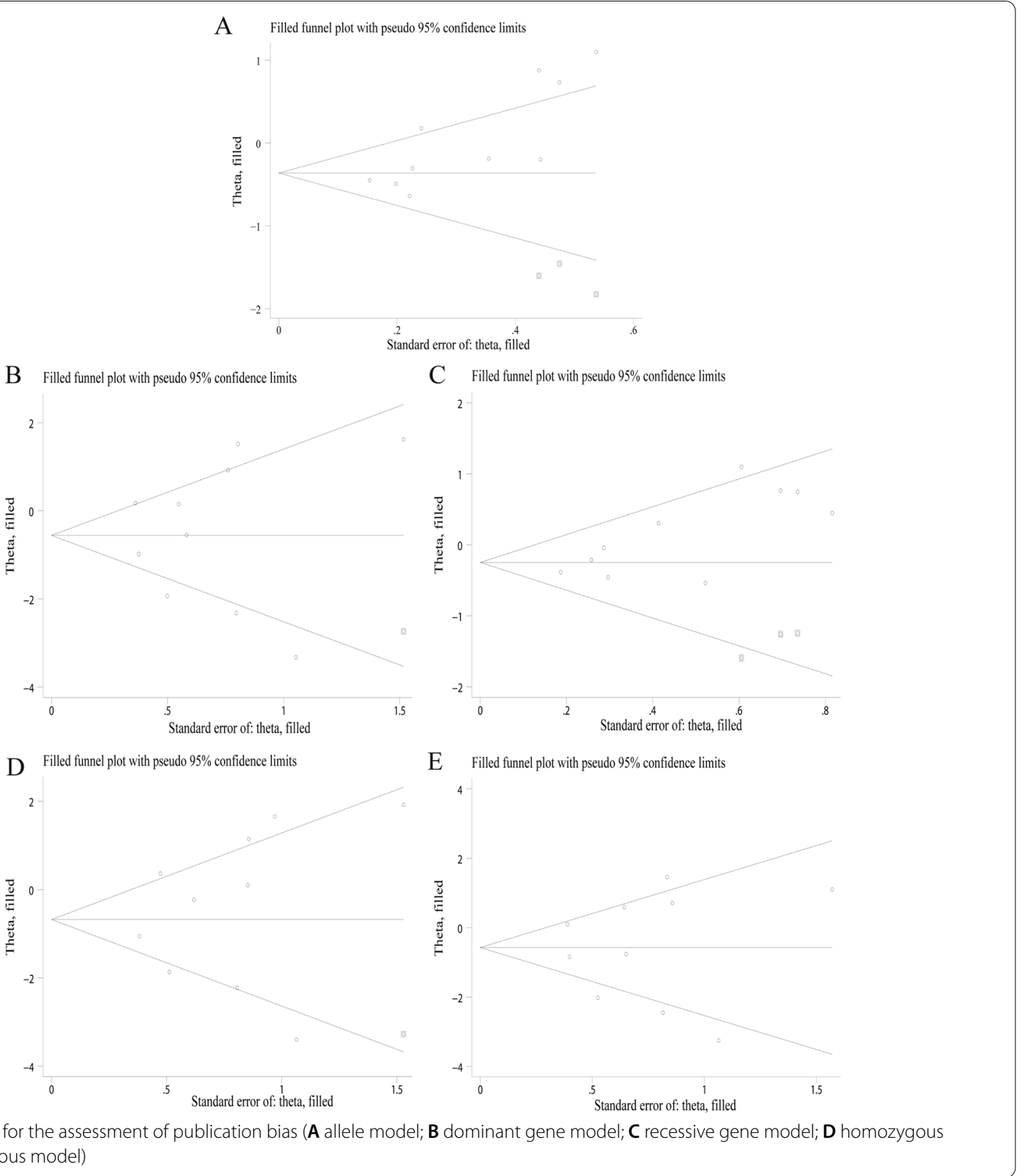

Fig. 3 Funnel plots for the assessment of publication bias (A allele model; $\mathbf{B}$ dominant gene model; $\mathbf{C}$ recessive gene model; $\mathbf{D}$ homozygous model; $\mathbf{E}$ heterozygous model)

difference was not statistically meaningful. The heterogeneity decreased apparently. Subgroup analysis of HWE showed that the decline in heterogeneity was not significant. The funnel plot was basically symmetrical (Fig. 3D), and Egger's Test showed that $P>0.05$, indicating that there was no publication bias.

\section{Heterozygous genetic model}

In the heterozygous genetic model (CT vs. TT), genotype $\mathrm{CT}$ was set as the exposure factor and genotype TT as the non-exposure factor. The results of the heterogeneity test showed that $\mathrm{I}^{2}=74.4 \%(P<0.05)$, indicating that there was a statistically significant difference in heterogeneity between studies. Therefore a REM was adopted. The forest plot was as shown in Fig. 2E. The results showed that $\mathrm{OR}=0.56(95 \% \mathrm{CI}: 0.25 \sim 1.27, P>0.05)$. The ethnic subgroup analysis showed that for the Asian population, $\mathrm{OR}=0.19$ (95\%CI:0.07 0.54), the difference was statistically meaningful. In the Caucasian population, the difference was not statistically noteworthy. The heterogeneity decreased substantially. While the subgroup analysis of HWE showed that the heterogeneity did not decrease. 
The funnel plot was basically symmetrical (Fig. 3E), and Egger's Test showed that $P>0.05$, which indicated that there no obvious publication bias.

\section{Sensitivity analysis}

In consideration of the correlation between ERCC1 rs11615 polymorphism and platinum chemosensitivity of ovarian cancer in the Asian population, a targeted sensitivity analysis in the Asian population was conducted (Fig. 4A-E). The results showed that in the allelic, dominant, recessive, and homozygous genetic models, when the number $4,1,3$, and 1 studies were eliminated, and the differences became insignificant. While in the heterozygous genetic model, with any study excluded, the conclusion did not change. As the above shows, there is a correlation between ERCC1 rs11615 polymorphism and platinum chemosensitivity of ovarian cancer in the Asian population. Meanwhile,

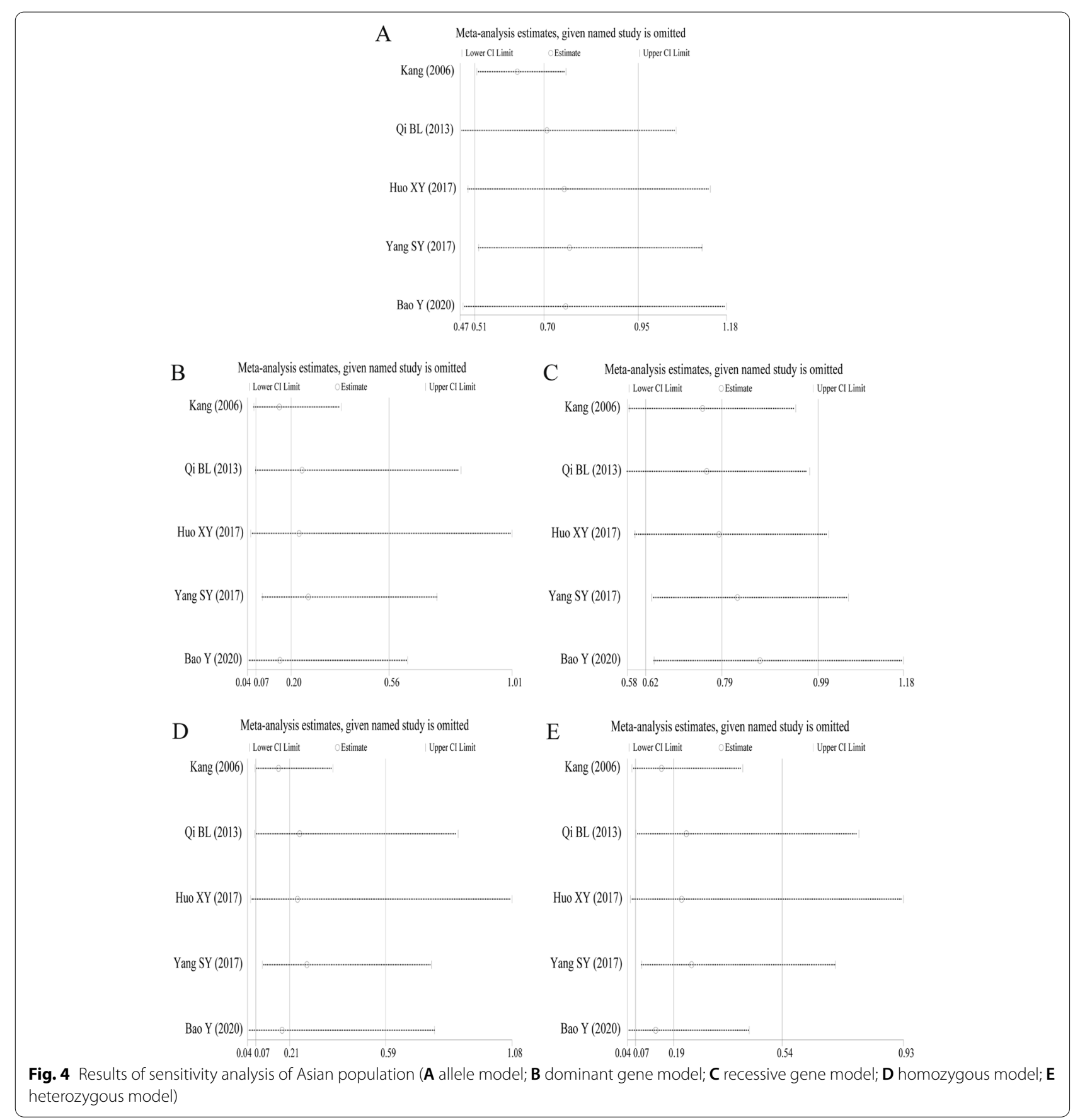


allele $\mathrm{C}$, genotype $\mathrm{CC}$, and $\mathrm{CT}$ can increase the sensitivity of ovarian cancer patients in the Asian population to platinum chemotherapy. However, it still needs careful interpretation in allele, dominant, recessive, and homozygous genetic models.

\section{Discussion}

At present, the conclusions upon the rs11615 polymorphism of the ERCC1 gene and the chemosensitivity of platinum drugs in ovarian cancer are not consistent. In order to explore the relationship between them, this meta-analysis was conducted, of which the results showed that there was a correlation between the rs11615 polymorphism of ERCC1 and the chemosensitivity of platinum drugs in ovarian cancer, mainly in the Asian population, but not in the Caucasian population. There are numerous factors that affect the sensitivity of chemotherapy, among which the polymorphism of ovarian oncogene and the chemosensitivity and clinical prognosis of platinum drugs have always been the focus of research [32, 33]. There are also plenty of studies on the relationship between ERCC1 gene polymorphism and platinum chemotherapy sensitivity in patients with ovarian cancer [21, 22, 24]. The ERCC1 gene is located on chromosome 9q13.2-q13.3, which is a DNA sequence containing 10 exons, with its length larger than $15 \mathrm{~kb}$ [34]. The gene encoding product is ERCC1 protein. In the NER pathway, ERCC1 protein and xeroderma pigmentosum complementation group $\mathrm{D}(\mathrm{XPD})$ together form a heterodimer of ERCC1-XPD, which is a $5^{\prime}-3^{\prime}$ DNA restricted endonuclease in NER, and also functions in DNA repair connection and inner chain cross repair [34]. These years, although studies have shown that overexpression of DNA repair genes can change the ability of DNA repair $[35,36]$, its regulatory mechanism is obscure yet. Some studies have suggested that single nucleotide polymorphism (SNP) can change the expression of repair genes [37-39], while other studies have shown that abnormally hypermethylation in the promoter region can inhibit gene expression, which will subsequently lead to the occurrence of some diseases and tumors and the resistance of chemotherapeutic drugs [40, 41]. ERCC1 gene is the dominant gene in NER and an important component of DNA damage identification and repair, of which the expression and single nucleotide polymorphism may affect platinum resistance and prognosis in patients with ovarian cancer [6, 42].

This meta-analysis eventually included ten studies with a total of 1866 patients with ovarian cancer. The results showed that the rs11615 polymorphism of the ERCC1 gene was strongly associated with ovarian cancer patients in the Asian population, and the differences were statistically important in allele, dominant, recessive, homozygous, and heterozygous genetic models. However, no such correlation was discovered in the Caucasian population. In accordance with the ethnicity subgroup analysis, whether in the Asian population or the Caucasian population, the heterogeneity declined significantly. A subgroup analysis was also conducted on whether the genotype frequency of the control group meets HWE, the results of which showed that according to the HWE subgroup analysis, the heterogeneity in each gene model did not notably decrease. The sensitivity analysis results in the Asian population showed that the conclusion in the heterozygous model was robust, but after the allelic, dominant, recessive, homozygous, and heterozygous genetic models were removed, the difference became not statistically significant. In each gene model, the funnel plot was approximately symmetrical. The $P$-value of Egger's Test in both allele model and recessive gene inheritance model was less than 0.05 , indicating that there was a certain publication bias in the two genetic models. As to other genetic models, the $P$ values of Egger's Test were all greater than 0.05 , which suggested that there was no significant publication bias in these genetic models. Consequently, it can be concluded that the rs11615 polymorphism of the ERCC1 gene is associated with ovarian cancer patients in the Asian population. Allele C, genotype CC, and CT will increase the sensitivity of ovarian cancer patients in the Asian population to platinum chemotherapy, but some genetic models still need to be explained carefully. Tang et al. [43] reported that there is no correlation between the rs11615 polymorphism of the ERCC1 gene and the chemosensitivity of platinum drugs in ovarian cancer, which is inconsistent with our conclusion. This may be for the reason that more upto-date and high-quality researches were included in this study. Li et al. [44] reported that the genotype TT at ERCC1 rs11615 locus increases the risk of death in patients with ovarian cancer after platinum chemotherapy, which is consistent with this study to some extent. A meta-analysis by Yang et al. in 2019 [45] showed that ERCC1 rs11615 polymorphism was not associated with chemosensitivity in patients with ovarian cancer, and a subgroup analysis of the Asian population showed the same conclusion. This is different from our conclusion. Our study showed that all genetic models were statistically significant in the Asian populations. Although only five studies were included in the analysis of Asian population, the sensitivity analysis confirmed the robustness of the conclusion in the heterozygote model. This has critical clinical significance, which not only points out a key direction for further research, but also provides a crucial basis for individualized treatment of ovarian cancer patients according to ERCC1 gene polymorphism in the future. 
At present, our study found that there is a correlation between ERCC1 rs11615 polymorphism and chemotherapy sensitivity of ovarian cancer in the Asian population, but not in the Caucasian population. It may be that there are differences between chemotherapy sensitivity and ovarian cancer patients of various ethnicity. It has been reported that several differences exist in chemotherapy sensitivity to gynecological tumors in different races [46]. In addition, it is possible that the included studies are limited, resulting in no differences in the Caucasian population.

Inevitably, this study is limited by the following factors. First, the number of literature included was relatively small. After ethnicity subgroup analysis, there were only five studies in the Asian population and the Caucasian population, respectively, which had a certain impact on the robustness of the conclusion. Second, the sample size was relatively small, which was likely to affect the testing efficiency of statistics. Third, there was a certain publication bias in allele model and recessive gene model. Last but not least, in the sensitivity analysis of the Asian population, only the conclusion of the heterozygous genetic model was stable, while the conclusion of other genetic models was unstable to a certain degree.

In conclusion, the ERCC1 rs11615 polymorphism is associated with the sensitivity of platinum-based combination chemotherapy in patients with ovarian cancer, especially in the Asian population. Allele $C$, genotype $\mathrm{CC}$, and CT will increase the sensitivity of ovarian cancer patients to platinum chemotherapy in the Asian population. Nevertheless, no such correlation has been found in the Caucasian population. Given that there are certain limitations in this study, such as small sample size and publication bias, the conclusions of this study still need to be further verified by larger sample size and high-quality clinical research.

\footnotetext{
Abbreviations

CNKI: China National Knowledge Infrastructure; NOS: Newcastle-Ottawa scale; REM: Random-effects model; FEM: Fixed-effects model; HWE: HardyWeinberg Equilibrium; OR: Odds ratio; NER: Nucleotide excision repair; XPD: Xeroderma pigmentosum complementation group D; SNP: Single nucleotide polymorphism.
}

\section{Acknowledgements}

None.

\section{Authors' contributions}

Yuqiang Zhang, Sufen Cao, Bailang Lin: Critical revision of the manuscript; Yuqiang Zhang, Sufen Cao, Chunyu Zhuang, Bailang Lin: Substantial contribution to the conception and design of the work, manuscript drafting;Yuqiang Zhang, Jiacheng Chen, Xiaojing Chen,Hong Sun, Shengying Lin: Acquisition, analysis, and interpretation of the data; Yuqiang Zhang,Sufen Cao,Chunyu Zhuang, Jiacheng Chen,Bailang Lin: Revising the manuscript critically, final approval of the version to be published. All authors have read and approved the final manuscript.
Funding

None.

\section{Availability of data and materials}

The datasets used and/or analysed during the current study are available from the corresponding author on reasonable request.

\section{Declarations}

Ethics approval and consent to participate

Ethical approval was not needed because this is a meta-analysis.

Consent for publication

Not applicable.

Competing interests

All the authors declare that they have no conflict of interest.

\section{Author details}

${ }^{1}$ Department of Obstetrics, Haikou Maternal and Child Health Hospital, Haikou, Hainan, China. ${ }^{2}$ Department of Nursing, Haikou Maternal and Child Health Hospital, No. 6 Wentan Road, Haikou 570203, Hainan, China. ${ }^{3}$ Department of Hepatological Surgery, Hainan Provincial People's Hospital, Haikou, Hainan, China. ${ }^{4}$ Department of Gynecology and Obstetrics, Haikou Maternal and Child Health Hospital, Haikou, Hainan, China. ${ }^{5}$ Department of Operation, Haikou Maternal and Child Health Hospital, Haikou, Hainan, China.

Received: 16 October 2020 Accepted: 28 May 2021

Published online: 21 June 2021

References

1. Bray F, Ferlay J, Soerjomataram I, Siegel RL, Torre LA, Jemal A. Global cancer statistics 2018: GLOBOCAN estimates of incidence and mortality worldwide for 36 cancers in 185 countries. CA Cancer J Clin. 2018;68(6):394-424

2. Kim S, Wang M, Tyrer JP, Jensen A, Wiensch A, Liu G, et al. A comprehensive gene-environment interaction analysis in ovarian cancer using genome-wide significant common variants. Int J Cancer. 2019;144(9):2192-205.

3. Zhang $H$, Zhou L. Single nucleotide polymorphism of PIK3CA and its interaction with the environment are risk factors for Chinese Han ovarian cancer. Pathol Res Pract. 2019;215(9):152520.

4. Friedlander M, Matulonis U, Gourley C, du Bois A, Vergote I, Rustin G, et al. Long-term efficacy, tolerability and overall survival in patients with platinum-sensitive, recurrent high-grade serous ovarian cancer treated with maintenance olaparib capsules following response to chemotherapy. Br J Cancer. 2018;119(9):1075-85.

5. Fang F, Cardenas H, Huang H, Jiang G, Perkins SM, Zhang C, et al. Genomic and epigenomic signatures in ovarian cancer associated with resensitization to platinum drugs. Cancer Res. 2018;78(3):631-44.

6. Zhang Z, Dou X, Yang H, Jia L, Qin K, Gao X, et al. Association of expression of p53, livin, ERCC1, BRCA1 and PARP1 in epithelial ovarian cancer tissue with drug resistance and prognosis. Pathol Res Pract. 2020;216(2):152794

7. Qin Y, Li W, Long Y, Zhan Z. Relationship between p-cofilin and cisplatin resistance in patients with ovarian cancer and the role of $p$-cofilin in prognosis. Cancer Biomark. 2019;24(4):469-75.

8. Wang X, Wang X, Guo Z. Functionalization of platinum complexes for biomedical applications. Acc Chem Res. 2015:48(9):2622-31.

9. Wang X, Guo Z. Targeting and delivery of platinum-based anticancer drugs. Chem Soc Rev. 2013;42(1):202-24.

10. Peng PJ, Cheng H, Ou XQ, Zeng LJ, Wu X, Liu YM, et al. Safety and efficacy of S-1 chemotherapy in recurrent and metastatic nasopharyngeal carcinoma patients after failure of platinum-based chemotherapy: multiinstitutional retrospective analysis. Drug Des Devel Ther. 2014;8:1083-7.

11. Wang YS, Tian J, Han Y, Han SM, Shi SB. Gemcitabine plus vinorelbine as second-line therapy in patients with metastatic esophageal cancer 
previously treated with platinum-based chemotherapy. Oncol Res. 2016;24(2):129-35.

12. Puppe J, Opdam M, Schouten PC, Jóźwiak K, Lips E, Severson T, et al. BRCA1EZH2 is overexpressed in -like breast tumors and predictive for sensitivity to high-dose platinum-based chemotherapy. Clin Cancer Res. 2019;25(14):4351-62.

13. Rottenberg S, Disler C, Perego P. The rediscovery of platinum-based cancer therapy. Nat Rev Cancer. 2021;21(1):37-50.

14. Novohradsky V, Zerzankova L, Stepankova J, Vrana O, Raveendran R, Gibson D, et al. New insights into the molecular and epigenetic effects of antitumor Pt(IV)-valproic acid conjugates in human ovarian cancer cells. Biochem Pharmacol. 2015;95(3):133-44.

15. Reed E. Platinum-DNA adduct, nucleotide excision repair and platinum based anti-cancer chemotherapy. Cancer Treat Rev. 1998;24(5):331-44.

16. Heinze $K$, Hölzer M, Ungelenk M, Gerth M, Thomale J, Heller R, et al. RUNX3 transcript variants have distinct roles in ovarian carcinoma and differently influence platinum sensitivity and angiogenesis. Cancers. 2021;13(3):476

17. Fong CW. Platinum anti-cancer drugs: free radical mechanism of PtDNA adduct formation and anti-neoplastic effect. Free Radic Biol Med. 2016;95:216-29.

18. Ishibashi M, Toyoshima M, Zhang X, Hasegawa-Minato J, Shigeta S, Usui $\mathrm{T}$, et al. Tyrosine kinase receptor TIE-1 mediates platinum resistance by promoting nucleotide excision repair in ovarian cancer. Sci Rep-Uk. 2018:8(1):13207

19. Colmegna B, Uboldi S, Frapolli R, Licandro SA, Panini N, Galmarini CM, et al. Increased sensitivity to platinum drugs of cancer cells with acquired resistance to trabectedin. Brit J Cancer. 2015;113(12):1687-93.

20. Yu JJ, Mu C, Lee KB, Okamoto A, Reed EL, Bostick-Bruton F, et al. A nucleotide polymorphism in ERCC1 in human ovarian cancer cell lines and tumor tissues. Mutat Res. 1997:382(1-2):13-20.

21. Steffensen $K$, Waldstrom $M$, Jeppesen $U$, Brandslund I, Jakobsen A. Prediction of response to chemotherapy by ERCC1 immunohistochemistry and ERCC1 polymorphism in ovarian cancer. Int J Gynecol Cancer. 2008;18(4):702-10.

22. Kang S, Ju W, Kim JW, Park NH, Song YS, Kim SC, et al. Association between excision repair cross-complementation group 1 polymorphism and clinical outcome of platinum-based chemotherapy in patients with epithelial ovarian cancer. Exp Mol Med. 2006;38(3):320-4.

23. Stang A. Critical evaluation of the Newcastle-Ottawa scale for the assessment of the quality of nonrandomized studies in meta-analyses. Eur J Epidemiol. 2010:25(9):603-5.

24. Smith S, Su D, Rigault DLLI, Schwartz P, Puopolo M, Rutherford TJ, et al. ERCC1 genotype and phenotype in epithelial ovarian cancer identify patients likely to benefit from paclitaxel treatment in addition to platinum-based therapy. J Clin Oncol. 2007;25(33):5172-9.

25. Steffensen KD, Waldstrøm M, Jakobsen A. DNA-repair ERCC1 gene polymorphisms in epithelial ovarian cancer and relation to platinum resistance and survival. J Cancer Ther. 2011;2(2):140-7.

26. Bosmuller H, Haitchi-Petnehazy S, Webersinke G, Marschon R, Roithmeier F, Stummvoll W, et al. Intratumoral lymphocyte density in serous ovarian carcinoma is superior to ERCC1 expression for predicting response to platinum-based therapy. Virchows Arch. 2011:459(2):183-91.

27. Qi BL, Li Y, Wang N, Zhou RM, Hu P, Kang S. Polymorphisms of ERCC1 gene and outcomes in epithelian ovarian cancer patients with platimumbased chemotherapy. Chin J Obstet Gynecol. 2013;48(11):847-52.

28. Huo XY, Xue J, Wang F, Wang J, Hao ZF. Association of RECC1 gene polymorphism with chemosensitivity and prognosis of TP for patients with epithelial ovarian. Anti Tumor Pharm. 2017;7(6):692-717.

29. Yang SY, Li Y, Kang S. Effect of ERCC1 gene polymorphisms on respond of platinum-based chemotherapy and prognosis in patients with epithelial ovarian cancer. Mod J Integr Tradit Chin West Med. 2017;26(11):1156-9.

30. Bao Y, Yang B, Zhao J, Shen S, Gao J. Role of common ERCC1 polymorphisms in cisplatin-resistant epithelial ovarian cancer patients: a study in Chinese cohort. Int J Immunogenet. 2020;47(5):443-53.
31. Moxley K, Benbrook D, Queimado L, Zuna R, Thompson D, Mccumber $M$, et al. The role of single nucleotide polymorphisms of the ERCC 1 and MMS19 genes in predicting platinum-sensitivity, progression-free and overall survival in advanced epithelial ovarian cancer. Gynecol Oncol. 2013;130(2):377-82

32. Khrunin A, Ivanova F, Moisseev A, Khokhrin D, Sleptsova Y, Gorbunova V, et al. Pharmacogenomics of cisplatin-based chemotherapy in ovarian cancer patients of different ethnic origins. Pharmacogenomics. 2012;13(2):171-8

33. Hu JL, Hu XL, Han Q, Guo AY, Wang CJ, Wen YY, et al. INSR gene polymorphisms correlate with sensitivity to platinum-based chemotherapy and prognosis in patients with epithelial ovarian cancer. Gene Ther. 2017:24(7):392-8.

34. Niedernhofer LJ, Odijk H, Budzowska M, van Drunen E, Maas A, Theil AF, et al. The structure-specific endonuclease Ercc1-Xpf is required to resolve DNA interstrand cross-link-induced double-strand breaks. Mol Cell Biol. 2004;24(13):5776-87.

35. Kuo IY, Huang YL, Lin CY, Lin CH, Chang WL, Lai WW, et al. SOX17 overexpression sensitizes chemoradiation response in esophageal cancer by transcriptional down-regulation of DNA repair and damage response genes. J Biomed Sci. 2019;26(1):20

36. Mazzu YZ, Armenia J, Chakraborty G, Yoshikawa Y, Coggins SA, Nandakumar S, et al. A novel mechanism driving poor-prognosis prostate cancer: overexpression of the DNA repair gene, ribonucleotide reductase small subunit M2 (RRM2). Clin Cancer Res. 2019;25(14):4480-92.

37. Zhao H, Kan Y, Wang X, Chen L, Ge P, Qian Z. Genetic polymorphism and transcriptional regulation of $C R E B B P$ gene in patient with diffuse large B-cell lymphoma. Biosci Rep. 2019;39(8):BSR20191162.

38. Malutan AM, Drugan C, Drugan T, Ciortea R, Mihu D. The association between interleukin-4-590C/T genetic polymorphism, IL-4 serum level, and advanced endometriosis. Cen Eur J Immunol. 2016:41(2):176-81.

39. El-Behedy EM, Akeel N, El-Maghraby HM, Shawky A. Serum level and genetic polymorphism of mannose-binding lectin in infants with neonatal sepsis at Zagazig University Hospitals. Egypt J Immunol. 2019:26(1):91-9.

40. Quinn JE, Carser JE, James CR, Kennedy RD, Harkin DP. BRCA1 and implications for response to chemotherapy in ovarian cancer. Gynecol Oncol. 2009:113(1):134-42

41. Livingston DM, Silver DP. Cancer: crossing over to drug resistance. Nature. 2008:451 (7182):1066-7.

42. Li FY, Ren XB, Xie XY, Zhang J. Meta-analysis of Excision Repair Crosscomplementation Group 1 (ERCC1) association with response to platinum-based chemotherapy in ovarian cancer. Asian Pac J Cancer Prev. 2013;14(12):7203-6.

43. Tang N, Lyu D, Zhang Y, Liu H. Association between the ERCC1 polymorphism and platinum-based chemotherapy effectiveness in ovarian cancer: a meta-analysis. BMC Womens Health. 2017;17(1):43.

44. Li Y, Yang SY, Kang S, Yip BH, Zhou RM, Wang N, et al. Association between polymorphisms of ERCC1 and survival in epithelial ovarian cancer patients with chemotherapy. Pharmacogenomics. 2012;13(4):419-27.

45. Yang F, Mu X, Bian C, Zhang H, Yi T, Zhao X, et al. Association of excision repair cross-complimentary group 1 gene polymorphisms with breast and ovarian cancer susceptibility. J Cell Biochem. 2019:120(9):15635-47.

46. Killelea BK, Yang VQ, Wang SY, Hayse B, Mougalian S, Horowitz NR, et al. Racial differences in the use and outcome of neoadjuvant chemotherapy for breast cancer: results from the National Cancer Data Base. J Clin Oncol. 2015;33(36):4267-76.

\section{Publisher's Note}

Springer Nature remains neutral with regard to jurisdictional claims in published maps and institutional affiliations. 